UNIVERSIDADE DE SÃO PAULO

FACULDADE DE ODONTOLOGIA DE RIBEIRÃO PRETO

\title{
ESTUDO DA RELAÇÃO ENTRE RESISTÊNCIA À ABRASÃO E DUREZA DE LIGAS ODONTOLÓGICAS DE METAIS BÁSICOS PARA RESTAURAÇÃO METALOCERÂMICA
}

\section{CELSO DE BARROS}

Tese apresentada à Faculdade de Odontologia de Ribeirão Preto da Universidade de São Paulo para obtenção do título de DOUTOR em Reabilitação Oral, junto ao Departamento de Materiais Dentários e Prótese.

\section{RIBEIRÃO PRETO}


UNIVERSIDADE DE SÃO PAULO

FACULDADE DE ODONTOLOGIA DE RIBEIRÃO PRETO

\section{AVALIAÇÃO DA RELAÇÃO ENTRE RESISTÊNCIA À ABRASÃO E DUREZA DE LIGAS ODONTOLÓGICAS DE METAIS BÁSICOS PARA RESTAURAÇÃO METALOCERÂMICA}

\section{CELSO DE BARROS}

Tese apresentada à Faculdade de Odontologia de Ribeirão Preto da Universidade de São Paulo para obtenção do título de DOUTOR em Reabilitação Oral, junto ao Departamento de Materiais Dentários e Prótese.

Orientador: Prof. Dr. Osvaldo Luiz Bezzon

\section{RIBEIRÃO PRETO}




\section{FICHA CATALOGRÁFICA}

\section{Preparada pela Biblioteca Central do Campus Administrativo de Ribeirão Preto / USP.}

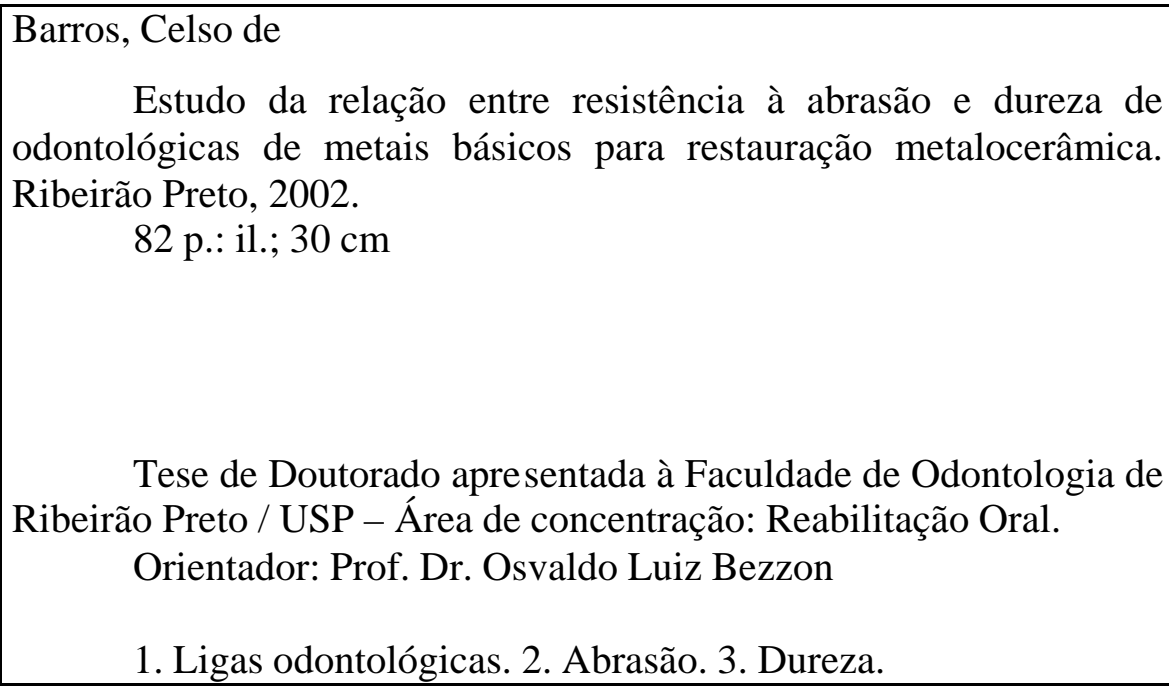

Tese de Doutorado apresentada à Faculdade de Odontologia de Ribeirão Preto / USP - Área de concentração: Reabilitação Oral.

Orientador: Prof. Dr. Osvaldo Luiz Bezzon

1. Ligas odontológicas. 2. Abrasão. 3. Dureza.

Endereço para correspondência:

Faculdade de Odontologia de Ribeirão Preto - USP

Departamento de Materiais Dentários e Prótese Av. do Café s/n - Monte Alegre

14040-904 - Ribeirão Preto - SP 


\section{CELSO DE BARROS}

Data da Defesa: 31/07/2002

\section{Banca Examinadora}

Prof. Dr. Osvaldo Luiz Bezzon (orientador) / Prof. Titular / Faculdade de Odontologia de Ribeirão Preto - Universidade de São Paulo.

Julgamento: Assinatura:

Prof. Dr. João Manuel Domingos de Almeida Rollo / Prof. Livre-docente / Escola de Engenharia de São Carlos - Universidade de São Paulo.

Julgamento: Assinatura:

Prof. Dr. Mário Fernando de Góes / Prof. Titular / Faculdade de Odontologia de Piracicaba / Universidade de Campinas.

Julgamento: Assinatura:

Prof. Dr. Carlos dos Reis Pereira de Araújo / Prof. Doutor / Faculdade de Odontologia de Bauru / Universidade de São Paulo.

Julgamento: Assinatura:

Profa. Dra. Iara Augusta Orsi / Profa. Doutora / Faculdade de Odontologia de Ribeirão Preto / Universidade de São Paulo.

Julgamento: Assinatura: 
"Quando nada parece ajudar eu vou e olho o cortador de pedras martelando uma rocha, talve $z$ cem vezes, sem que nenhuma rachadura apareça. No entanto, na centésima primeira martelada, a pedra se abre em duas e eu sei que não foi somente aquela que conseguiu, mas todas as que vieram antes".

\section{Por isSo, DEDICO ESTE TRABALHO:}

aos meus pais, gosé Cândido e Diva, pelo esforço, apoio, carinfo e incentivo constantes que me proporcionaram transpor as dificuldades e chegar neste estágio da profissão;

e à minfra doce e que rida avó Elvira, pela discrição de seus gestos, em toda a vida, fazendo-me compreender a sobreposição da fumildade perante o orgulho. 
O homem atrás do balcão olhava a rua de forma distraída. Uma garotinha aproximou-se da loja e a massou o narizinho contra o vidro da vitrine. Os olhos da cor do céu brilhavam quando viu um deteminado objeto. Entrou na loja e pediu para ver o colar de turquesa azul.

- É para minha imã. Pode fazer um pacote bem bonito?, disse ela.

O dono da loja olhou desconfiado para a garotinha e lhe perguntou:

- Quanto dinheiro você tem?

Sem hesitar, ela tirou da saia um lenço todo amarradinho e foi desfazendo os nós. Colocou-o sobre o balcão e feliz, disse:

- Isso dá?

Eram apenas algumas moedasque ela exibia orgulhosa.

- Sabe, quero dar este presente para minha imã mais velha. Desde que morreu nossa mãe, ela cuida da gente e não tem tempo para ela. É aniversá rio dela e tenho certeza que fic ará feliz com o colarque é da corde seusolhos.

O homem foi para o interior da loja, colocou o colar em um estojo, embrulhou com vistoso papelvermelho e fez um laço caprichado com uma fita verde.

- Tome, disse para a garota. Leve-o com cuidado.

Ela saiu feliz, saltitando pela rua abaixo.

Ainda não acabara o dia qua ndo uma linda jovem de cabelos loiros e maravilhosos olhos azuis entrou na loja. Colocou sobre o balcão o já conhecido embrulho desfeito e indagou:

- Este colar foi compra do aqui?

- Sim, senhora.

- Equanto custou?

- Ah! Falou o dono da loja. O preço de qualquer produto da minha loja é sempre um assunto confidencial entre o vendedore o cliente.

A moça continuou:

“Mas minha imã tinha apenas algumas moedas! O colar é verdadeiro, não é? Ela não teria dinheiro para pagá-lo!"

O homem tomou o estojo, refez o embrulho com extremo carinho, colocou a fita e o devolveu à jovem.

- Ela pagou o preço mais alto que qualquer pessoa pode pagar. ELA DEU TUDO O QUETINHA.

O silêncio encheu a pequena loja e duas lágrimas rolaram pela face emocionada da jovem enquanto suas mãos tomavam o pequeno embrulho.

Verdadeira doação é dar-se por inteiro, sem restrições. Gratidão não coloca limites para os gestos de temura. Seja sempre grato, mas não espere pelo reconhecimento de ninguém. Gratidão não a penas aquece quem recebe, como reconforta quem oferece.

\section{MUTIO OBRIGADO,}

Ao prof. Dr. João Manuel Domingos de Almeida Rollo, pelo incentivo e orientação constantes, fundamentais para a realização deste trabalho.

Aos Srs. Pedro Luiz di Lorenzo e José Silvano Cerqueira Lima, técnico de laboratório do Departamento de Materiais da Escola de Engenharia de São Carlos da Universidade de São Paulo, pelo valioso apoio técnico durante toda a pesquisa. 
Ao Sr. José Godói Filho, técnico de laboratório do Departamento de Materiais Dentários e Prótese da FORP-USP, pela participação na obtenção dos corpos-de-prova de titânio.

Ao laboratório ART DONT, sob a administração do Sr. César Simões Azenha, pela atenção e participação direta na confecção dos corpos-de-prova das ligas de Ni-Cr e Co-Cr.

Ao Sr Amauri da Costa Vicari, funcionário da oficina da prefeitura do Campus Administrativo de Ribeirão Preto - USP, pela expressiva colaboração na retificação dos padrões de nylon utilizados no processo de fundição dos corpos-de-prova.

À $C N G$, sob a administração do engenheiro Sr. Luiz Fernando Buratto, pela atenção e informações, importantes para o enriquecimento do trabalho.

Aos profs. Drs. Geraldo Maia Campos e Iara Augusta Orsi, pela atenção e importante orientação na realização da análise estatística.

Ao Sr. Marcelo Pessolo dos Santos pela, valiosa atenção dedicada na formatação deste trabalho.

Aos profs. do Departamento de Materiais Dentários e Prótese da FORP - USP, pela atenção despendida na convivência diária.

Aos profs. vinculados ao programa de Pós-graduação na área de Reabilitação Oral da FORP -USP, pela orientação e apoio.

À Sra. Maria Cristina M. Ferreira, funcionária da Biblioteca Central da Prefeitura do Campus Administrativo de Ribeirão Preto - USP, pela valiosa disponibilidade, atenção e responsabilidade dedicada na revisão do texto.

À profa. Laudenete F. Ramalho, pelas valiosas sugestões na correção ortográfica do texto.

À Isabel Cristina G. Sola e Regiane Cristina Moi Sacilotto, funcionárias da secretaria da seção de Pós-graduação da Faculdade de Odontologia de Ribeirão Preto - USP, pela atenção e paciência despendidas.

À Regiane de Cássia Tirado Damasceno e Maria Tereza de Freitas Iossi, funcionárias do departamento de Materiais Dentários e Prótese pela atenção e paciência despendidas.

À profa. Dra. Lauralice de Campos Franceschini Canale, pela disponibilidade do laboratório, sob sua responsabilidade, para a realização dos testes de abrasão.

À Data Net Informática, administrada pelos Srs. Marcos Valério Rufim e Edgard Rufim Jr., pelo apoio técnico despendido. 
Aos professores, membros titulares e suplentes, pelas valiosas sugestões na composição final do texto.

Ao $C N P Q$, por financiar parte do trabalho (proc. n. 144785/1998-6).

\section{Prof. Dr. Osvaldo Luiz Bezzon}

\section{PROCURA-SE}

Procura-se um amigo. Nãa precisa ser fromem, Gasta ser fumano, basta ter sentimento, basta ter coração. Precis a saber falar e calar, sobretudo saber ouvir o que as palavras não dizem. Ter que gostar de poesia, de madrugada, de pássaros, das estrelas, do sol, da lua, do canto dos ventos e das canções da brisa.

Deve ter amor, um grande amor por alguém, ou então sentir falta de não ter esse amor. Deve amar o próximo e respeitar a dor que os passantes levam consigo. Deve guardar segredo sem se sacrificar. Nãa é preciso que seja de primeira mão, nem é imprescindivel que seja de segunda mão. Pode já ter sido enganado, pois todos os amigos são enganados. Nãa é preciso que seja puro, nem que seja de todo impuro, mas não deve ser vulgar. Deve ter um ideal e medo de perdê-lo e, no caso de assim não ser, deve sentir o grande vácuo que is so de ixa.

Tem de ter ressonâncias humanas, seu principal objetivo deve ser o de amigo. Deve sentir pena das pessoas tristes e compreender o imenso vazio dos solitários. Deve gostar de crianças e lastimar as que não puderam nascer.

Procura-se um amigo para gostar dos mesmos gostos, que se comova quando chamado de amigo. Que saiba conversar de coisas simples, de orvalho, de grandes chuvas e das recordações da infância.

Preciso de um amigo para não enlouquecer, para contar o que vi de belo e triste durante o dia, dos anseios e das realizações, dos sonthos e da realidade.

Deve gostar de ruas desertas, de poças d'água e de caminhos malhados de beira de estrada, de mato de pois da chuva, de se deitar no capim.

Preciso de um amigo que diga que vale a pena viver, não porque a vida é bela, mas porque já tenfo um amigo.

Preciso de um amigo para parar de chorar. Para não viver de bruçado no passado em busca de memórias perdidas.

Que Gata nos ombros sorrindo e chorando, mas que me chame de amigo, para que eu tenha a consciência de que ainda vivo. 
Obrigado pela orientação, atenção e confiança.

Você foi mais do que um orientador. Você foi um AMIGO.

Serei sempre grato!!!

Este trabalho foi realizado nas seguintes instituições:

- Departamento de Materiais Dentários e Prótese da Faculdade de Odontologia de Ribeirão Preto da Universidade de São Paulo.

- Departamento de Materiais da Escola de Engenharia de São Carlos da Universidade de São Paulo. 
SUMÁRIO

LISTA DE ABREVIATURAS E SIGLAS

LISTA DE SÍMBOLOS

RESUMO

ABSTRACT

1. INTRODUÇÃO 01

2. REVISÃO DA LITERATURA 04

3. PROPOSIÇÃO 44

4. MATERIAL E MÉTODO 45

4.1. Ligas metálicas 45

4.2.1. Obtenção dos corpos de prova 46

4.2. Ensaios 47

4.2.1. Ensaio de abrasão 47

4.2.2. Determinação dos valores de dureza 49

4.2.3. Determinação do peso (gramas) 49

4.3. Análise metalográfica 49

4.4. Análise estatística 50

5. RESULTADOS 52

6. DISCUSSÃO 64

7. CONCLUSÕES 72

8. ANEXOS 73

9. REFERÊNCIAS BIBLIOGRÁFICAS 78

10. APÊNDICE 
LISTA DE ABREVIATURAS E SIGLAS

\begin{tabular}{cc}
\hline Abreviatura e sigla & Significado \\
\hline Et al. & e colaboradores \\
\hline $\mathrm{Kg}$ & Quilograma \\
$1 \mathrm{Kgf}$ & 1 Quilograma-força $=9,807$ Newtons \\
$1 \mathrm{Libra}$ & 453,6 gramas \\
$\mathrm{mm}$ & Milímetro \\
$\mathrm{m}$ & Metro \\
$1 \mathrm{mega}$ & $10^{6}$ \\
$\mathrm{ml}$ & Mililitro \\
$\mathrm{min}$ & Minuto \\
$\mathrm{MOD}$ & Mésio-ocluso-distal \\
$\mathrm{Mpa}=\mathrm{MN} / \mathrm{m}^{2}$ & Megapascal = Mega-Newton $/ \mathrm{m}^{2}$ \\
$\mathrm{seg}$ & Segundo \\
$\mu \mathrm{m}=10^{-6} \mathrm{~m}=10^{-3} \mathrm{~m}$ & micrômetro \\
$\mathrm{ISO}$ & International Organization Standard \\
$\mathrm{ADA}$ & American Dental Association \\
\hline
\end{tabular}




\section{LISTA DE SÍMBOLOS}

\begin{tabular}{|c|c|}
\hline Símbolo & Significado \\
\hline$\%$ & Por cento \\
\hline${ }^{\circ} \mathrm{C}$ & Grau Celsius \\
\hline$>$ & Maior \\
\hline$<$ & Menor \\
\hline$=$ & Igual \\
\hline$\#$ & Granulação \\
\hline $\mathrm{Al}$ & Alumínio \\
\hline Bal & Complemento para $100 \%$ \\
\hline $\mathrm{Be}$ & Berílio \\
\hline $\mathrm{C}$ & Carbono \\
\hline $\mathrm{Cd}$ & Cádmio \\
\hline Co & Cobalto \\
\hline $\mathrm{Cr}$ & Cromo \\
\hline $\mathrm{Fe}$ & $\mathrm{Fe}$ \\
\hline $\mathrm{g}$ & Grama (s) \\
\hline $\mathrm{Ga}$ & Gálio \\
\hline $\mathrm{H}$ & Hidrogênio \\
\hline $\mathrm{H}_{2} \mathrm{O}$ & Água \\
\hline Ir & Irídio \\
\hline $\mathrm{Mg}$ & Magnésio \\
\hline $\mathrm{Mn}$ & Manganês \\
\hline Mo & Molibdênio \\
\hline $\mathrm{N}$ & Nitrogênio \\
\hline $\mathrm{Nb}$ & Nióbio \\
\hline $\mathrm{Ni}$ & Níquel \\
\hline $\mathrm{O}$ & Oxigênio \\
\hline $\mathrm{P}$ & Fósforo \\
\hline $\mathrm{Pd}$ & Paládio \\
\hline $\mathrm{Pl}$ & Platina \\
\hline $\mathrm{S}$ & Enxofre \\
\hline $\mathrm{Si}$ & Silício \\
\hline $\mathrm{Sn}$ & Estanho \\
\hline $\mathrm{W}$ & Tungstênio \\
\hline $\mathrm{Ti}$ & Titânio \\
\hline $\mathrm{V}$ & Vanádio \\
\hline $\mathrm{Zn}$ & Zinco \\
\hline
\end{tabular}




\section{RESUMO}

Barros, C. Estudo da relação entre resistência à abrasão e dureza de ligas odontológicas de metais básicos para restauração metalocerâmica. 2002. 82p. Tese Doutorado - Faculdade de Odontologia de Ribeirão Preto, Universidade de São Paulo, Ribeirão Preto.

Embora haja grande exigência por restaurações estéticas livres de metal, restaurações metalocerâmicas, cujas subestruturas feitas com ligas de metais básicos, são procedimentos de rotina nos planejamentos restauradores protéticos. No entanto, a alta resistência ao desgaste destas ligas, caracterizada pela grande dificuldade de acabamento e polimento, tem sido associada à dureza, geralmente elevada. A proposta deste estudo foi avaliar resistência à abrasão e dureza de seis ligas de metais básicos (4 ligas de $\mathrm{Ni}-\mathrm{Cr}, 1$ liga de $\mathrm{Co}-\mathrm{Cr}$ e $1 \mathrm{Ti}$ comercialmente puro - 99,5\% Ti) disponíveis comercialmente para uso com cerâmica. A resistência à abrasão foi determinada pela perda de peso dos corposde-prova, ao longo de 4 séries de ciclos de abrasão. A dureza Rockwell 30N também foi avaliada nos mesmos intervalos. Os dados foram analisados com o nível de significância $\alpha$ $=0,05$. O teste de regressão e correlação da resistência à abrasão e dureza mostrou que para 3 ligas de Ni-Cr (Vera Bond, Vera Bond II e Wiron 99) a linha de regressão foi descendente, indicando que um aumento na dureza correspondeu a uma redução nos valores de perda de peso, enquanto para 1 liga de $\mathrm{Ni}-\mathrm{Cr}$ (Neochrom) a linha de regressão foi ascendente, indicando um aumento nos valores de perda de peso, apesar de um aumento na dureza. Por outro lado, para a ligas de Co-Cr (New Ceram) e de titânio (Rematitan), os valores de perda de peso se mantiveram constantes. As ligas avaliadas tiveram baixos coeficientes de correlação entre resistência à abrasão e dureza. A análise metalográfica revelou particularidades que podem ter sido determinantes para as taxas de desgaste de cada material. Com exceção da liga de titânio, não houve mudanças metalográficas das ligas avaliadas, durante as séries de ciclos de abrasão.

Palavra-chave: Ligas Odontológicas, Abrasão, Dureza 


\begin{abstract}
Barros, C. Study of relationship between abrasion resistance and hardness of dental base metal alloys for metal ceramic restoration. 2002. 82p. Tese Doutorado - Faculdade de Odontologia de Ribeirão Preto, Universidade de São Paulo, Ribeirão Preto.
\end{abstract}

In spite of the demand for metal free restorations, metal ceramic restorations using base metal alloys are routine in prosthodontics. Nevertheless, the high abrasion resistance of base metal alloys, characterized by difficulty in finishing and polishing, has been related to excessive hardness. The purpose of this study was to evaluate abrasion resistance and hardness of six base metal alloys (4 Ni-Cr alloys, $1 \mathrm{Co}-\mathrm{Cr}$ alloy and 1 commercially pure $\mathrm{Ti}$ alloy $-99.5 \% \mathrm{Ti}$ ) available commercially for use with fused porcelain. Abrasion resistance was determined by specimen weight loss along 4 series of abrasion cycles. The Rockwell $30 \mathrm{~N}$ hardness was also determined in the same periods. Data were analyzed statistically with the level of significance set at $\alpha=0.05$. Regression and correlation analysis of abrasion resistance and hardness showed that for three Ni-Cr alloys (Vera Bond, Vera Bond II and Wiron 99) the regression lines descended indicating that an increase in hardness corresponded to a reduction in weight loss, whereas for one Ni-Cr alloy (Neochrom) the regression line ascended indicating an increase in weight loss in spite of an increase in hardness. On the other hand, for the Co-Cr alloy (New Ceram) and Ti (Rematitan), the weight loss remained constant. The alloys tested had low correlation coefficients between abrasion resistance and hardness. Microscopic analysis showed details that may have been determining for the weight loss rate of each material. With the exception of the titanium alloy, there were no metallographic changes of the alloys tested during the abrasion tests.

Key-words: Dental Alloys, Abrasion, Hardness 


\section{INTRODUÇÃO}

Restaurações ou reposições de superfícies mastigatórias são procedimentos que têm como objetivo o tratamento de várias condições patológicas bucais (MAHALICK et al., 1971).

Embora haja grande exigência por restaurações estéticas livres de meta e conseqüente desenvolvimento de técnicas e materiais inovadores, restaurações com subestruturas metálicas, principalmente as metalocerâmicas, ainda são procedimentos de rotina nos planejamentos restauradores protéticos.

O desenvolvimento de coroas e próteses fixas metalocerâmicas iniciou-se a partir da década de 50. Desde então, as tradicionais ligas de metais nobres para próteses fixas foram substituídas por não apresentarem propriedades físicas e mecânicas necessárias para uso com cerâmica. Assim, concomitante aos avanços na área das cerâmicas, houve a introdução de novas ligas de ouro que, durante muitos anos, foram predominantemente usadas para confecção de subestruturas metálicas de restaurações metalocerâmicas (O'BRIEN et al., 1964).

A partir da década de 70, o ouro teve aumento significativo no custo (MOFFA et al., 1973a; WANG \& LI, 1998). Como resultado, estudos foram conduzidos no sentido de desenvolver ligas de metais básicos, de custo relativamente baixo, como material alternativo às ligas de ouro.

Evidentemente o alto custo do ouro, por si, não justificaria o uso de materiais restauradores de qualidade inferior. Na realidade, contribuiu para incrementar o número de pesquisas por novos materiais, o que proporcionou o desenvolvimento de ligas de metais básicos, também chamadas alternativas, com propriedades físicas e mecânicas 
comparáveis às das ligas de metais nobres, possibilitando situações clínicas bastante favoráveis.

A ampla diferença de densidade, somada ao alto custo do ouro por unidade de peso, foi motivo ainda maior para implementar o uso destes novos materiais tanto que, em 1981, a American Dental Association constatou que as ligas de Ni-Cr e CoCr já eram usadas por $70 \%$ dos laboratórios e representavam quantidade considerável de trabalhos de prótese fixa (OLIN et al., 1989).

$\mathrm{Na}$ década de 80, o titânio também surgiu como material protético alternativo ao ouro (KING et al., 1994). A excelente biocompatibilidade do titânio, associada às adequadas propriedades químicas, físicas e mecânicas (TAÍRA et al., 1989), e a marcante evolução na tecnologia de fundição, têm estimulado seu uso para confecção de subestruturas de restaurações fixas e removíveis. Apesar da exigência de um processo metalúrgico de produção sofisticado, o titânio ainda é considerado um material de baixo custo.

Embora, hoje em dia, países desenvolvidos estejam estimulando o retorno ao uso de ligas nobres na confecção de subestruturas para restaurações metalocerâmicas, no Brasil, o contato com profissionais da área mostra que as ligas de metais básicos, principalmente de $\mathrm{Ni}-\mathrm{Cr}$ e $\mathrm{Co}-\mathrm{Cr}$, apesar de não serem universalmente aceitas (WANG \& LI, 1998) pelo caráter citotóxico de alguns elementos metálicos presentes na sua composição (KELLY \& ROSE, 1983; BEZZON, 1993), predominam sobre as demais, sendo o uso do titânio ainda incipiente.

A alta resistência ao desgaste das ligas de metais básicos tem sido associada à dureza, geralmente elevada (CIVJAN et al., 1972; KING et al., 1994). Uma restauração com maior valor de dureza tende manter a superfície polida por mais tempo devido à sua alta resistência ao risco, mas é mais difícil para ajuste e acabamento superficial (MOON \& MADJESKI, 1976; MORRIS, 1989, 1990; KING et al., 1994).

Há um conceito popularmente mantido de que a resistência ao desgaste de um material pode ser prevista pelas características de resistência mecânica e dureza (HARRISON \& DRAUGHN, 1976; BARAN, 1983). Estudos mostraram, entretanto, que pode não haver relação direta entre resistência, dureza e desgaste abrasivo (Moser \& Greener (1972, 1973) apud HARRISON \& DRAUGHN, 1976). BEZZON et al. (2001) 
concluíram que o uso de valores de dureza para estimar a resistência à abrasão de uma liga pode acarretar um conceito errôneo.

É comum a abrasividade ser diretamente relacionada à dureza (JACOBI et al., 1991). No entanto, o estudo da taxa de desgaste abrasivo é um mecanismo complexo (HARRISON \& DRAUGHN, 1976; DRAUGHN \& HARRISON, 1978; ANUSAVICE, 1996). Portanto, relacionar dureza superficial diretamente com maior ou menor dificuldade de usinagem de ligas odontológicas de metais básicos necessita de especial atenção, que será objetivo deste estudo. 